\title{
Continuous Dynamic Mapping of the Corticospinal Tract in Motor Eloquent Tumor Surgery: Our Experience and Evaluation of the Method
}

\author{
Andrej Porčnik ${ }^{1}$, Jure Pešak ${ }^{1}$, Tilen Žele ${ }^{1}$, Blaž Koritnik ${ }^{2}$, Zoran Rodi ${ }^{2}$, Borut Prestor ${ }^{1}$ \\ ${ }^{1}$ Department of Neurosurgery, University Medical Centre Ljubljana, Slovenia, ${ }^{2}$ Institute of Clinical Neurophysiology, \\ University Medical Centre Ljubljana, Slovenia
}

Correspondence: andrej.porcnik@kclj.si; Tel.: +386 31773 527; Fax.: + 3865225129

Received: 19 May 2020; Accepted: 11 October 2020

\begin{abstract}
Objective. The aim of this article is to present our experience with continuous dynamic mapping (CDM) of the corticospinal tract (CST) when removing tumors in motor eloquent regions. Methods. We studied 44 patients with a brain tumor adjacent to the CST where CDM was used. The mapping probe was integrated at the tip of the suction device. Thresholds for eliciting MEPs were recorded. In all patients, along with CDM, MEPs to direct cortical stimulation were also monitored throughout the operation. Motor function was assessed preoperatively, after the procedure and on discharge. Results. In the series, there were 37 patients with gliomas, six with brain metastasis, and one with cavernoma. The threshold to elicit MEPs in CDM was $>20$ $\mathrm{mA}$ in 17 cases, $16-20 \mathrm{~mA}$ in six cases, $11-15 \mathrm{~mA}$ in six cases, $6-10 \mathrm{~mA}$ in nine cases and 2-5 $\mathrm{mA}$ in six cases. MEPs to direct cortical stimulation were preserved in all patients. In three cases a new temporary motor deficit was noted. No new permanent motor deficit occurred. Gross total resection was reached in $57 \%$ of cases. Conclusions. From our experience, the combined use of CDM and MEPs to direct cortical stimulation improves the safety of surgery in the proximity of the CST, and at the same time offers the possibility of higher rates of gross total resection.
\end{abstract}

Key Words: Subcortical Mapping - Corticospinal Tract • Tumor Surgery • Motor Evoked Potential • Intraoperative Monitoring.

\section{Introduction}

Operating in the proximity of the corticospinal tract (CST) represents a challenge to every surgeon, i.e., removing as much of the tumor tissue as possible while ensuring the preservation of motor function. There is increasing evidence that maximizing the extent of the resection improves survival in low and high grade gliomas (1-4)the prognostic significance of eloquent brain tumor location and the role of functional mapping during resective surgery in presumed eloquent brain regions are unknown. Methods. We performed a retrospective analysis of 281 cases involving adults who underwent resection of a supratentorial LGG at a brain tumor referral center. Preoperative MR images were evaluated blindly for involvement of eloquent brain areas, including the sensorimo- tor and language cortices, and specific subcortical structures. For high-risk tumors located in presumed eloquent brain areas, long-term survival estimates were evaluated for patients who underwent intraoperative functional mapping with electrocortical stimulation and for those who did not. Results. One hundred and seventy-four patients $(62 \%$. On the other hand, better postoperative performance status without new motor deficits has been shown to be an independent prognostic factor (5).

The assistance of intraoperative neurophysiological techniques is proving invaluable in such circumstances. Traditionally, to evaluate the integrity of the CST, motor evoked potentials (MEPs) are used, elicited by transcranial electrical stimulation, and detected as electromyographic (EMG) responses in the muscles of the upper and lower limbs. In the case of brain tumors, as the brain is 
exposed, MEPs are best elicited by direct cortical or subcortical stimulation. Strategically, neurophysiological intervention may be viewed as either mapping or monitoring. Mapping may begin with stimulation of the cortex and recording MEPs in the muscles of the face, hands and legs, primarily to divide eloquent motor areas from non-eloquent, and to find the optimal point of entry into the tissue. After that, a stationary electrode may be positioned over the eloquent area of the cortex to enable continuous monitoring of MEPs during surgery. Mapping may then continue subcortically with stimulation of the walls of the emerging cavity throughout the removal of the tumor, thus enabling the estimation of the proximity of the CST.

In addition to intermittent and punctiform mapping, a new technique called "continuous dynamic mapping" (CDM) was introduced in 2014 (6-8). The mapping probe is integrated into the tip of a suction device which enables continuous stimulation, while the EMG responses in the corresponding facial muscles and muscles of the upper and lower limbs are detected in order to evaluate the proximity of the CST. In comparison to older techniques, CDM gives more precise temporal and spatial coverage of the surgical site, and better real time feedback of the location of the CST during tumor removal $(6,8,9)$.

Knowing the distance from the CST provided by the CDM, and the integrity of the CST provided by monitoring through direct cortical stimulation, offers the possibility of more radical tumor resection, and at the same time the preservation of motor function.

In this article we present our experience with these methods in a series of patients from our own clinical practice.

\section{Methods}

\section{Patient Population}

We conducted a prospective study on 44 patients with brain tumors located less than $20 \mathrm{~mm}$ away from the CST (measured on MRI) in whom CDM and monitoring were used intraoperatively. Clinical and neurological parameters were evaluated preoperatively. The size and location of the tumor was assessed on the basis of preoperative magnetic resonance imaging (MRI). To estimate the size of the tumor, we measured the volume of hyperintense areas in FLAIR sequence for Grade II gliomas and the volume of contrast-enhancement in T1 sequence for Grade III and IV gliomas. All patients underwent tumor resection at the Department of Neurosurgery of the University Medical Centre, Ljubljana, between December 2016 and March 2020. All patients signed informed consent.

\section{Intraoperative Data and the Use of Dynamic Cortical Mapping During Tumor Removal}

We performed either awake brain surgery (11 patients) or surgery under general anesthesia (33 patients). Neuronavigation was used in all cases. Additionally, 5-aminolevulinic acid (5-ALA) was used in all high grade glioma cases.

The intraoperative neurophysiological approach consisted of monitoring and CDM, using the Nicolet Viasys Endeavor system (Figure 1). Both monitoring and mapping utilized an EMG signal recorded by pairs of monopolar needle electrodes, inserted into standard target muscles of interest, namely the $\mathrm{m}$. orbicularis oris, $\mathrm{m}$. abductor digiti minimi and $\mathrm{m}$. tibialis anterior. For monitoring, anodal stimulus was applied to the cortical surface using a strip electrode placed on the precentral gyrus, and for mapping, cathodal stimulus was applied subcortically using the mapping probe integrated into the tip of a suction device. In both monitoring and mapping, monopolar stimulation was used with the other pole being a corkscrew electrode placed on the nearby scalp. A short train stimulus was used, consisting of four pulses, each pulse of $0.5 \mathrm{~ms}$ pulse duration, with an interpulse interval of $4 \mathrm{~ms}$, frequency of $2 \mathrm{~Hz}$ between the trains, and stimulation intensity up to $20 \mathrm{~mA}$. Anesthesia was induced and maintained with propofol and remifentanil, and a bolus of short acting relaxant was applied for the purpose of intubation $(6,9,10)$

CDM during tumor removal started at $15-20 \mathrm{~mA}$. The appearance of EMG responses was observed at a sensitivity of 100 microvolts per unit. When a re- 

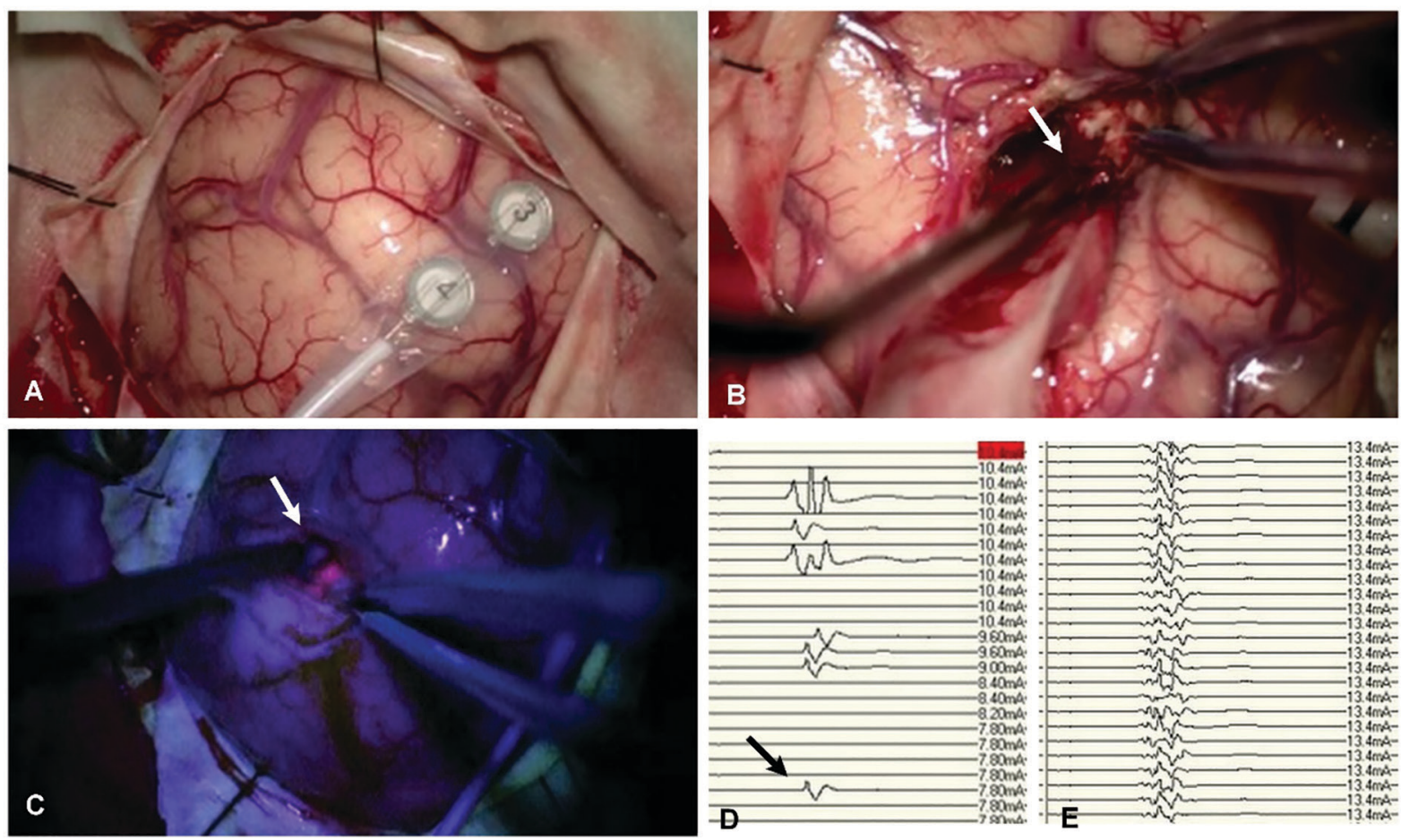

Figure 1. For direct cortical stimulation for MEP monitoring, a strip electrode is placed over the motor cortex (A). For subcortical continuous dynamic mapping, the mapping probe is integrated into the tip of a suction device (white arrow) - shown under white (B) and blue light (C). During tumor resection, the motor thresholds provided by mapping (D) and direct cortical (E) MEPs are monitored. In this case, the lowest motor threshold was $7.8 \mathrm{~mA}$ (black arrow).

peatable EMG response appeared, the resection was stopped, and the stimulus intensity was reduced in $2 \mathrm{~mA}$ steps, until the response disappeared, and the tumor removal continued. When $5 \mathrm{~mA}$ was reached, stimulus was reduced in $1 \mathrm{~mA}$ steps. Resection was stopped at 3-5 $\mathrm{mA}$. If there was the possibility of total tumor removal, resection was continued, but not below $2 \mathrm{~mA}$. The final lowest stimulation amplitude (in $\mathrm{mA}$ ) needed to evoke an EMG response was noted as the lowest motor threshold.

During the whole procedure, the EMG responses from direct cortical stimulation were monitored. A 50\% or more decrease in amplitude of the MEPs was considered meaningful. If this occurred, the resection was paused for 15 minutes and stopped if the decrease persisted after the 15-minute pause.

\section{Postoperative Data}

Within 48 hours after surgery MRI was performed in glioma patients, and MRI or CT in patients who underwent surgery for metastasis. The extent of resection was calculated as the difference between the preoperative and postoperative tumor volume. It was classified according to the proportion of the resected volume as: gross total resection (resection of the whole volume), subtotal resection (at least $90 \%$ of the volume) or reduction (less than $90 \%$ of the volume). Postoperative clinical evaluation was performed one day after the operation and on the day of discharge from the hospital. Pathohistological diagnosis was established and complications were noted. Data are presented as absolute value, proportion (\%) or mean \pm standard deviation.

\section{Results}

\section{Patient Characteristics}

We included 44 patients, of whom 30 were men and 14 were women (Table 1). The mean age was $53 \pm 17$ years. The brain tumor was newly diag- 
nosed in 42 patients, and two patients were suffering from recurrence. The most common presenting symptoms were epileptic seizures and hemiparesis. The mean preoperative Karnofsky Performance Scale was $88 \pm 11$.

\section{Perioperative Findings}

Gliomas were present in $84 \%$ of patients, and metastatic or vascular lesions in $16 \%$. Data on pathohistological diagnosis are presented in Table 1.

All procedures were technically successful. Gross total resection was achieved in 57\%, subto-

Table 1. Characteristics of Presented Cases

\begin{tabular}{ll}
\hline Characteristic & Number of patients \\
\hline Gender male/female & $30 / 14$ \\
\hline Presenting symptom & \\
\hline Epileptic seizure & 24 \\
\hline Hemiparesis & 14 \\
\hline Cognitive decline & 3 \\
\hline Memory deficit & 1 \\
\hline Headache & 1 \\
\hline Dysphasia & 1 \\
\hline Pathohistological diagnosis & \\
\hline Glioma WHO IV & 22 \\
\hline Glioma WHO III & 7 \\
\hline Glioma WHO II & 8 \\
\hline Metastasis & 6 \\
\hline Cavernous angioma & 1 \\
\hline Extent of resection & 3 \\
\hline Gross total resection & 25 \\
\hline Subtotal resection & 10 \\
\hline Reduction & 9 \\
\hline Lowest motor threshold & 6 \\
\hline >20 mA & 9 \\
\hline $16-20$ mA & \\
\hline $11-15$ mA & \\
\hline $6-10$ mA & \\
\hline $2-5$ mA & \\
\hline New postoperative motor deficit & \\
\hline Temporary & \\
\hline Permanent & \\
\hline
\end{tabular}

tal resection in $23 \%$ and reduction in 20\% (Figure 2). In 19 patients, gross total resection was not achieved. In 16 of those, partial resection was already planned preoperatively because of the infiltration of other eloquent structures, such as the basal ganglia, thalamus, optic radiation, speech areas etc. In the remaining three patients the resection was stopped at the motor threshold of 3-5 $\mathrm{mA}$, after we recognized that complete resection was not possible. The lowest motor thresholds reached are presented in Table 1 . The amplitude of the MEPs remained stable or was reduced by less than $50 \%$ throughout the whole operation in all cases.

\section{Postoperative Complications}

New postoperative motor deficit was noted in $7 \%$ of patients $(\mathrm{N}=3)$ on the first postoperative day. On the day of discharge, the postoperatively acquired deficits had reversed in all cases. There was one case of intracerebral hematoma and one case of postoperative abscess, which both needed reoperation. Neither patient presented with neurological deficits on discharge. One patient with metastasis died in the postoperative period due to sepsis, and one patient suffered from a pulmonary embolism.

\section{Discussion}

Every physician should aspire to offer his patients as much as possible and constantly strive to improve the results of their work. In our opinion, an optimal result in tumor surgery close to the CST cannot be achieved with surgical techniques alone. Methods that provide information on the location of the tumor and eloquent motor regions, and the integrity of the CST have already proved helpful. With their use, we can improve our results beyond those achieved by even the most accomplished surgical technique.

To localize relevant structures, neuronavigation and, in some centers, even intraoperative MRI can be used. Additionally, 5-ALA can be used to localize high grade gliomas. The disadvantage 

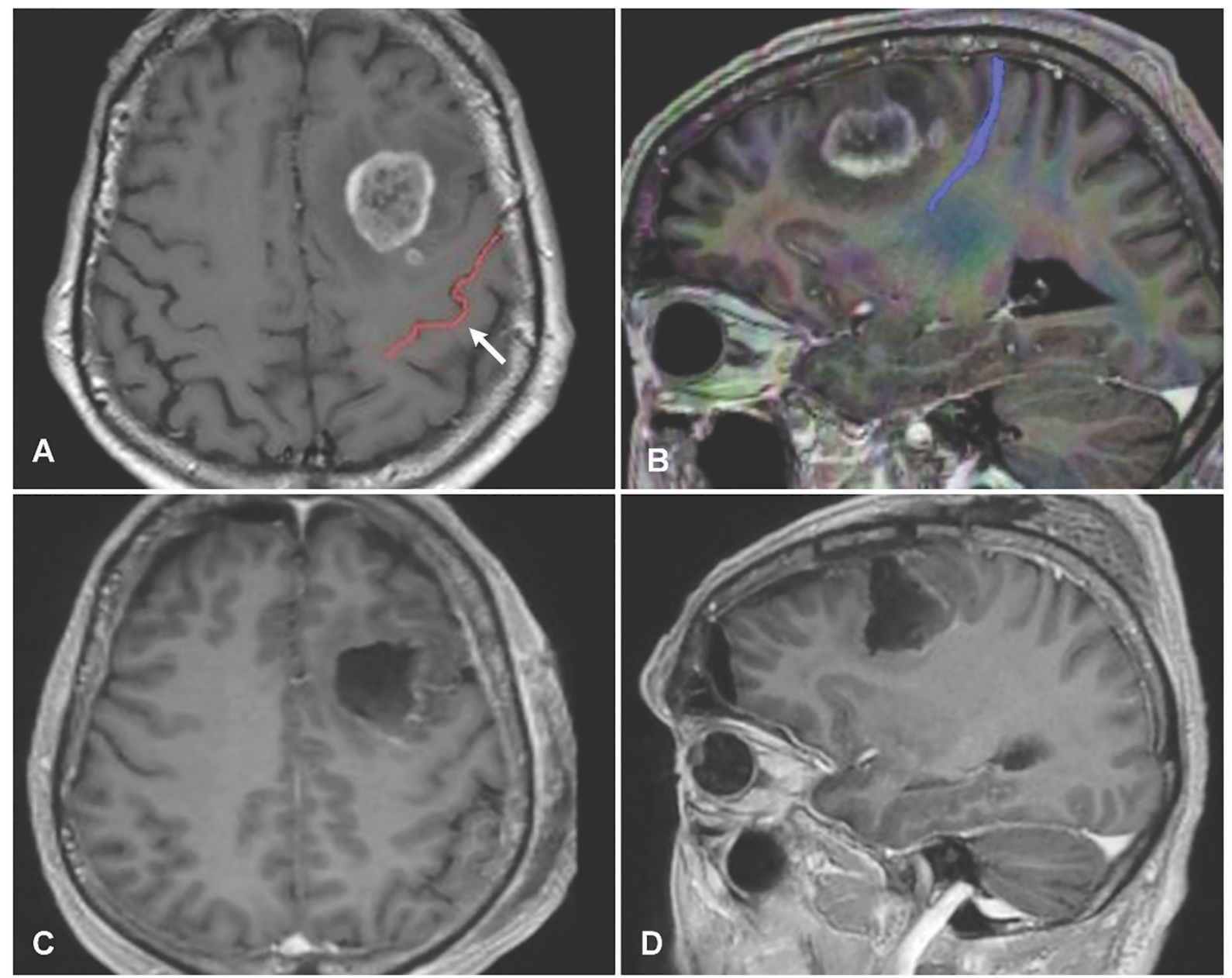

Figure 2. Preoperative contrast-enhanced MRI (A) and tractography (B) of a patient with a glioblastoma in the left precentral area. The arrow indicates the central sulcus. Tractography $(B)$ shows the corticospinal tract less than $10 \mathrm{~mm}$ away from the tumor. Postoperative MRI (C, D) reveals gross total resection (C, D). No new postoperative motor deficit was noted.

of neuronavigation is that after the release of the cerebrospinal fluid, and during removal of the tumor, the brain shifts and the method is no longer so precise. Intraoperative MRI is not widely available, it is time consuming, and it does not offer continuous feedback. Furthermore, 5-ALA fluorescent tissue may surpass the area of contrast enhancement on preoperative MRI by up to 10 $\mathrm{mm}$ (11). Fluorescence guided surgery can therefore lead to greater resection than the preoperative MRI shows. Moreover, all these methods only provide location information and do not test the function of eloquent motor regions. On the other hand, combined neurophysiological techniques of mapping and monitoring offer information on both. Therefore, we chose this approach to prevent possible CST damage during tumor removal.

Mapping was performed using a stimulation system integrated into the suction device, and it continuously provided information on the distance between the CST and the exact point of tumor removal (6-8). In assessing the distance to the CST, the rule of thumb, mainly derived from the results of intermittent mapping, is that " $1 \mathrm{~mA}$ correlates to $1 \mathrm{~mm}$ " $(6-8,12,13)$. The application of this rule to the CDM during tumor surgery is also supposed to be safe, however it has not yet been studied extensively $(6,7,9,11)$.

In our series, the lowest motor threshold reached at the end of tumor removal was below 
$6 \mathrm{~mA}$ in $14 \%$ and below $11 \mathrm{~mA}$ in $34 \%$. No new permanent motor deficit occurred in our series, however, we must acknowledge that the number of patients included was small. In previous studies with the use of intermittent subcortical mapping, a new permanent motor deficit was noted in $3.5-17 \%$ of patients, and it was more common when the lowest motor threshold was below $5 \mathrm{~mA}$ $(9,13-17)$ in an operation theater equipped with an integrated high-field intraoperative magnetic resonance imaging and a neuronavigation system. Diffusion-tensor imaging-based tractography of the CST was implemented preoperatively and intraoperatively. When the surgically manipulated area came close to the corticospinal pathway, MEP responses were elicited by subcortical stimulation. Responsive areas were compared with the locations of fibers traced by preoperative and intraoperative tractography. Imaging and functional outcomes were reviewed. Results Intraoperative tractography demonstrated significant inward or outward shift during surgery. MEP responses were observed around the tract at various intensities, and the distance between MEP responsive sites and intraoperative tractography was significantly correlated with the stimulation intensity $(\mathrm{P}<0.01)$. The use of CDM stimulation permitted even more radical tumor resection, with motor thresholds as low as $1-3 \mathrm{~mA}$, and new permanent motor deficit in $1.7-3 \%$ of patients $(6,9)$. According to a previous study, permanent motor worsening is mainly due to vascular injury and not so much to mechanical injury (6).

In our series, three patients presented with a new temporary motor deficit. The lowest motor threshold was below $11 \mathrm{~mA}$ in all three of them. This is in accordance with other studies, where either temporary or permanent motor deficits usually occurred below $11 \mathrm{~mA}(6,7,9)$. In another report, temporary motor deficit was present in 30\% of patients, which is more than was noted in our series (6). The lowest motor threshold in that report was below $5 \mathrm{~mA}$ in $59 \%$ of patients. Therefore, we assume that the distance from the tumor to the CST was greater in our series, which may explain the difference in the lowest motor threshold and the rate of motor deficit. However, we cannot exclude the possibility of a more radical approach in tumor removal in that report.

To evaluate the integrity of the CST, we used direct cortical monitoring throughout the operation. The alteration in the amplitude of MEPs was a warning sign of impending CST damage, and led us to consider pausing or stopping the resection, even in amplitude reductions smaller than 50\%. Gross total resection was achieved in $57 \%$ of cases. Similar previous studies reported gross total resection in approximately $70 \%$ of patients $(6,8,13,14)$. These results are difficult to compare because of the differences in tumor types, and their size and distance from the CST.

In our opinion, mapping and monitoring enable us to operate safely in the proximity of the CST. They proved to be useful in different kind of tumors. In cases of metastases and cavernomas, which are generally well-defined, they still influenced our resection strategy, tissue manipulation and hemostasis technique. Some metastases can be very adherent to the white matter, but in our series all were well-defined, and gross total resection was achieved in all cases. On the other hand, in gliomas, which are infiltrative tumors, mapping and monitoring are of the utmost importance in preventing damage to the CST by tumor removal. In order to operate without interruption, Raabe et all. proposed the use of a sound alert system that alerts us when the subcortical EMG response is elicited by the suction device (6). In our practice, continuous feedback was provided by a clinical neurophysiologist.

\section{Conclusion}

From our experience, the combined use of subcortical CDM and monitoring of cortically elicited MEPs improves the safety of operations in the proximity of the CST, and offers the possibility of higher rates of gross total resection. It provides confidence to the team participating in the procedure, and improves patient outcome and satisfaction. 


\section{What Is Already Known on this Topic:}

The rate of resection and preservation of motor function are known prognostic factors for the survival of patients with brain tumors. Therefore, the aim of tumor surgery in the proximity of the corticospinal tract (CST) is to remove as much tumor tissue as possible, while ensuring the preservation of motor function. The assistance of intraoperative neurophysiological techniques is proving invaluable in such circumstances. Continuous dynamic mapping (CDM) was introduced is 2014, and its use is related to a higher rate of gross total resection and a higher rate of preservation of motor function in some studies.

\section{What this Study Adds:}

We show that the combined use of subcortical CDM and monitoring of cortically elicited motor evoked potentials resulted in a satisfactory level of safety of surgery in the proximity of the CST, and offered the possibility of higher rates of gross total resection.

Authors' Contributions: Conception and design: AP, JP and ZR; Acquisition, analysis and interpretation of data: AP, JP, TŽ and ZR; Drafting the article AP and JP; Revising it critically for important intellectual content: ZR, BK and BP; Approved final version of the manuscript: AP, JP, ZR, BK and BP.

Conflict of Interest: The authors declare that they have no conflict of interest.

\section{References}

1. Chang EF, Clark A, Smith JS, Polley MY, Chang SM, Barbaro NM, et al. Functional mapping-guided resection of low-grade gliomas in eloquent areas of the brain: Improvement of long-term survival. J Neurosurg. 2011;114(3):566-73.

2. Jakola AS, Myrmel KS, Kloster R, Torp SH, Lindal S, Unsgård G, et al. Comparison of a Strategy Favoring Early Surgical Resection vs a Strategy Favoring Watchful Waiting in Low-Grade Gliomas. JAMA. 2012;308(18):1881.

3. Sanai N, Polley MY, McDermott MW, Parsa AT, Berger MS. An extent of resection threshold for newly diagnosed glioblastomas. J Neurosurg. 2011;115(1):3-8.

4. Lacroix M, Abi-Said D, Fourney DR, Gokaslan ZL, Shi W, DeMonte F, et al. A multivariate analysis of 416 patients with glioblastoma multiforme: prognosis, extent of resection, and survival. J Neurosurg. 2001;95(2):190-8.

5. Liang J, Lv X, Lu C, Ye X, Chen X, Fu J, et al. Prognostic factors of patients with Gliomas- A $n$ analysis on 335 patients with Glioblastoma and other forms of Gliomas. BMC Cancer. 2020;20(1):35.

6. Raabe A, Beck J, Schucht P, Seidel K. Continuous dynamic mapping of the corticospinal tract during surgery of mo- tor eloquent brain tumors: evaluation of a new method. J Neurosurg. 2014;120(5):1015-24.

7. Schucht P, Seidel K, Jilch A, Beck J, Raabe A. A review of monopolar motor mapping and a comprehensive guide to continuous dynamic motor mapping for resection of motor eloquent brain tumors. Neurochirurgie. 2017;63(3):175-80.

8. Seidel K, Schucht P, Beck J, Raabe A. Continuous Dynamic Mapping to Preserve the Corticospinal Tract during Surgery of Motor Eloquent Brain Tumors. Klin Neurophysiol. 2019;50(4):220-6.

9. Seidel K, Beck J, Stieglitz L, Schucht P, Raabe A. The warning-sign hierarchy between quantitative subcortical motor mapping and continuous motor evoked potential monitoring during resection of supratentorial brain tumors. J Neurosurg. 2013;118(2):287-96.

10. Deletis V, Fernández-Conejero I. Intraoperative monitoring and mapping of the functional integrity of the brainstem. J Clin Neurol. 2016;12(3):262-73.

11. Schucht P, Knittel S, Slotboom J, Seidel K, Murek M, Jilch A, et al. 5-ALA complete resections go beyond MR contrast enhancement: shift corrected volumetric analysis of the extent of resection in surgery for glioblastoma. Acta Neurochir (Wien). 2014;156(2):305-12.

12. Shiban E, Krieg SM, Haller B, Buchmann N, Obermueller T, Boeckh-Behrens T, et al. Intraoperative subcortical motor evoked potential stimulation: how close is the corticospinal tract? J Neurosurg. 2015;123(3):711-20.

13. Maesawa S, Fujii M, Nakahara N, Watanabe T, Wakabayashi $\mathrm{T}$, Yoshida J. Intraoperative tractography and motor evoked potential (MEP) monitoring in surgery for Gliomas around the corticospinal tract. World Neurosurg. 2010;74(1):153-61.

14. Prabhu SS, Gasco J, Tummala S, Weinberg JS, Rao G. Intraoperative magnetic resonance imaging-guided tractography with integrated monopolar subcortical functional mapping for resection of brain tumors. J Neurosurg. 2011;114(3):719-26.

15. Duffau H. Stimulation mapping of white matter tracts to study brain functional connectivity. Nat Rev Neurol. 2015;11(5):255-65.

16. Gil Robles S, Gatignol P, Capelle L, Mitchell M-C, Duffau $\mathrm{H}$. The role of dominant striatum in language: a study using intraoperative electrical stimulations. J Neurol Neurosurg Psychiatry. 2005;76(7):940-6.

17. Duffau H. Contribution of intraoperative electrical stimulations in surgery of low grade gliomas: a comparative study between two series without (1985-96) and with (1996-2003) functional mapping in the same institution. J Neurol Neurosurg Psychiatry. 2005;76(6):845-51. 